VOL. I (1969) 385-389.

\title{
On algebraic rings
}

\section{Chacron}

A ring $R$ is $\pi$-regular (periodic) if for each 'element $x$ of $R$ there is $n=n(x)$ so that $x^{n}=x^{n} \cdot a \cdot x^{n}\left(x^{n}=x^{n} \cdot 1 \cdot x^{n}\right)$ (a depending on $x$ ). Let $R$ be an algebraic algebra over a commutative ring $F$ with identity. In this paper we prove that if every $\pi$-regular image of the ring $F$ is periodic, then $R$ is periodic. This result applies in particular to the algebraic rings $R$ (over the integers) considered by Drazin and to the algebraic algebras $R$ over algebraically prime fields. It extends a result of Drazin on torsin-free algebraic rings and a generalization by this author of Drazin's result.

In [5, Cor. 3.1] Drazin proved that if $R$ is a torsion-free ring which is algebraic over the integers, then $R$ is a nil ring. In [3, Prop. 2] we proved that every ring $R$ which is algebraic over the integers, must in fact be a periodic ring (i.e., $\forall x \in R \exists n>m \geq 1, x^{n}=x^{m} \quad[1$, Introduction]). Since a periodic ring can be characterized as a ring such that given $x$, we can find $n=n(x)$ such that $x^{n}$ generates a finite subring $[1$, p. 6 and 3, Prop. 1], it is clear that Drazin's result is a particular case of [3, Prop. 2]. In [5, Th. 5.6] (and in other well-known papers) rings $R$ algebraic over finite fields or, more generally, over periodic fields were considered. It is therefore natural to seek a generalization of [3, Prop. 2] to algebraic algebras $R$ over arbitrary commutative rings $F$ with $I$ (in the sense of Drazin ${ }^{1}$ ) with $F$ extending

Received $18 \mathrm{July} 1969$. This research has been partly supported by Grant A4 807 of the NRC of Cansds and by a summer fellowship of the Canadian Mathematical Congress.

1 However, in this paper we let the elements of $F$ be pure 'scalars' of the algebra $A$. Thus $A$ might be annihilated by non-zero scalars of $F$. 385 
suitably the case of the integers and that of the periodic rings. In this paper we prove that if the ring $F$ satisfies the condition that

(c) every $\pi$-regular homomorphic image of $F$ is periodic, then every algebra algebraic over $F$ must in fact be periodic.

Since every $\pi$-regular image of the integers is finite, hence periodic, we see that our result contains as particular case [3, Prop. 2].

If $R$ is an algebraic algebra with the property (c), say a (c)-algebra, then each idempotent element $e$ of $R$ will generate a locally finite subring $F . e$ (i.e., every finitely generated subring of $F . e$ is finite). In fact, $F . e$ is a subalgebra of $R$ ring homomorphic to $F$. Since $F . e$ is algebraic, $F . e$ is $\pi$-regular. By (c), F.e is periodic. Since $F$ has a unit and is commutative, $F . e$ is a unitary commutative periodic ring, hence $F . e$ is locally finite. (This result was shown in [4, Th. 2] in the more general case of a periodic ring with 1 satisfying a polynomial identity.) We have proved the following

LEMMA. If $R$ is a (c)-algebra, then each of its cyclic subalgebras generated by an idempotent is locally finite (as a ring).

We are now in a position to prove the

THEOREM. Every (c)-azgebra $R$ is periodic.

Notations. For $a \in R,[a](\langle a\rangle)$ denotes the multiplicative subsemigroup (subring) of $R$ generated by $a$.

Proof. We have to prove that $[a]$ is finite for all $a \in R$, which will be certainly the case for any nilpotent element $a$ of $R$. Assume that $a$ is non-nilpotent. We can find a polynomial $p(t)$ in one indeterminate having as coefficients elements of $F$ such that $a^{m}=p(a) \cdot a^{m+1}$. We may assume (without loss of generality) that $p(t)$ is a non-constant polynomial without constant term, whence $p(a) \in R$. By standard computation

$$
a^{m}=p(a) \cdot a \cdot a^{m}=p^{2}(a) a^{2} \cdot a^{m}=\ldots=a^{m} \cdot p^{m}(a) \cdot a^{m},
$$

so, for $e=p^{m}(a) \cdot a^{m}$, we have

$$
0 \neq e=e^{2}=p^{m}(a) \cdot a^{m}, \quad a^{m} \cdot e=e \cdot a^{m}=a^{m} .
$$


Set: $R_{e}=e \cdot R \cdot e ; b=a e=e a$. We have $p(a e)=p(a) . e$ so that

$$
e=b^{m} \cdot p^{m}(b), b^{m}=a^{m} \text { and } b^{m}=p(b) \cdot b^{m+1} \text {. }
$$

From $b \in R_{e}$ and $e=b^{m} \cdot p^{m}(b)$, we derive that $b$ is an invertible element of the ring $R_{e}$. Then $b^{m}=p(b) \cdot b^{m+1}$ yields $e=p(b) \cdot b$, which is to say that the inverse $c$ of $b$ in $R_{e}$ is precisely $p(b)$; in symbols :

$$
c=b^{-1}=p(b)=\alpha_{1} b+\ldots+\alpha_{k} b^{k}
$$

for some $\alpha_{1}, \ldots, \alpha_{k} \in F$. By multiplying both sides of the equality by $b^{-k-1}$ we obtain an equality of the form

$$
c^{k+2}=\alpha_{k} c+\ldots+\alpha_{1} c^{k} .
$$

By standard computation

$$
[c] \subseteq \sum_{j=1}^{k} A \cdot c^{j}
$$

where $A$ stands for the subring of $F$ generated by the set $\left\{\alpha_{1}, \ldots, \alpha_{k}\right\}$. By the Lemma, $F . e$ is locally finite. It follows that the subring $\left\langle\alpha_{j} e\right\rangle_{j}$ generated by the set $\left\{\alpha_{1}, e, \ldots, \alpha_{k}, e\right\}$ is finite. Clearly, $\left\langle\alpha_{j} \cdot e\right\rangle_{j}=\left\langle\alpha_{j}\right\rangle_{e}=A . e$. Therefore A.e is finite, a fortiori $A . c^{j}=(A . e) c^{j}$ is finite, whence $\sum_{j} A . c^{j}$ is finite. All in all we have proved that [c] is finite, which, combined with the property for $c$ to possess the inverse $b$ in $R_{e}$, tells us that $b^{2}=c^{2}=e$ for some $2 \geq 1$.

From $b^{m}=a^{m}$, follows $e=e^{2}=e^{m}=b^{2 m}=b^{m l}=a^{m l} \in[a]$, proving thereby that [a] is finite; this for every non-nilpotent element $a$ of $R$, and $R$ is periodic.

COROLLARY 1. Let $R$ be a ring. The following conditions are equivalent. 
(i) $R$ is algebraic over the integers.

(ii) $R$ is periodic.

(iii) $\forall x \in R \exists n, m \geq 1,\left(x^{n+1}-x\right)^{m}=0$.

(iv) For some two-sided ideal $A$ of $R, R / A$ and $A$ are periodic.

This Corollary is an immediate consequence of the Theorem. It tells us by standard argumentation that if $R$ is an arbitrary ring, one can define a maximal periodic ideal $L$ such that $R / L$ is periodic-simple (i.e., it has no non-zero periodic ideals). Also, by (iii), every periodic ring $R$ is a quasi-radical extension of the subring generated by the nilpotent elements of $R$. From [7] follows immediately

COROLLARY 2. Every (c)-algebra having all its nilpotent elements central is commutative.

This Corollary extends [5, Th. 5.5]. We note that in [5, Th. 5.5] or [6, Th.], the authors used [8, Th.], which is more general than [7]. The following extends [5, Th. 5.6].

COROLLARY 3. Let $F$ be a commutative ring with 1 . Let $R$ be an algebraic algebra over $F$. Assume that $F$ is algebraic over its prime subring 〈I〉. If, further, all nilpotent elements of $R$ are central, then $R$ is commutative.

Proof. Clearly $F$ is a (c)-algebra with respect to its subring (1). Therefore, $F$ is periodic (Theorem). Consequently, $R$ is a (c)-algebra, whence periodic. By Corollary 2, $R$ is commutative.

REMARK. By a general property of periodic rings [2, Th. 9], if $R$ is an algebra as in Corollary 3 , its subdirect irreducible components $A$ are local rings. Also, $R$, modulo its prime radical, is a ring in which every element $x$ satisfies $x=x^{n+1}$, for some $n \geq 1$ depending on $x$.

\section{References}

[1] M. Chacron, "Certains anneaux périodiques", Bulz. Soc. Math. Belg. 20 (1968), 66-77.

[2] M. Chacron, "On quasi periodic rings", J. Aigebra 12 (1969), 49-60. 
[3] M. Chacron, "On a theorem of Herstein", Canad. J. Math. (to appear).

[4] M. Chacron, "On a theorem of Procesi", submitted to J. Algebra.

[5] M.P. Drazin, "Algebraic and diagonable rings", Canad. J. Math. 8 (1956), $341-354$.

[6] I.N. Herstein, "A note on rings with central nilpotent elements", Proc. Amer. Math. Soc. 5 (1954), 620.

[7] I.N. Herstein, "A generalization of a theorem of Jacobson, III", Amer. J. Math. 75 (1953), 105-111.

[8] I.N. Herstein, "The structure of a certain class of rings", Amer. $J$. Math. 75 (1953), 866-871.

University of Windsor,

windsor, Ontario,

and

University of British Columbia,

Vancouver, British Columbia. 